\title{
Treatment of background in $\gamma-\gamma$ fast-timing measurements
}

\author{
E. R. Gamba ${ }^{\mathrm{a}, *}$, A. M. Bruce ${ }^{\mathrm{a}}$, M. Rudigier ${ }^{\mathrm{b}}$ \\ ${ }^{a}$ School of Computing Engineering and Mathematics, University of Brighton, Brighton BN2 \\ 4 GJ, United Kingdom \\ ${ }^{b}$ Department of Physics, University of Surrey, Guildford GU2 7XH, United Kingdom
}

\begin{abstract}
Background characterization in $\gamma-\gamma$ fast-timing measurements is of essential importance when lifetimes of the order of tens of picoseconds are being measured. In this work, the nature, composition and behaviour of the timing background is extensively discussed and an adaptation of the background subtraction method used in $\mathrm{E}_{\gamma}-\mathrm{E}_{\gamma}-\mathrm{E}_{\gamma}$ cubes to the case of $\mathrm{E}_{\gamma}-\mathrm{E}_{\gamma}-\Delta \mathrm{T}$ cubes, is presented. This is applied to ${ }^{252} \mathrm{Cf}$ fission data, showing very low peak-tobackground ratios (less than 0.5), collected using a hybrid array made of 51 HPGe detectors from Gammasphere and $25 \mathrm{LaBr}_{3}(\mathrm{Ce})$ scintillators. Two different procedures are suggested: the "Interpolation" approach and the "Three Samples" approach. Both were used to measure the lifetime of the $2^{+}$state in ${ }^{110} \mathrm{Ru}$, and gave $\tau=483(38)$ ps and $\tau=445(34) \mathrm{ps}$, respectively, both within one standard deviation of the literature value of $\tau_{l}=462(29) \mathrm{ps}$. The $2^{+}$state in ${ }^{114} \mathrm{Pd}$ was also measured using the three samples approach and the lifetime obtained was $\tau=104(12) \mathrm{ps}$, consistent with the literature lifetime of $\tau_{l}=118(20) \mathrm{ps}$.
\end{abstract}

Keywords: Fast-timing, $\gamma-\gamma$ coincidences, Lifetime measurements, Background correction, Centroid shift method, GCD method

\footnotetext{
${ }^{*}$ Corresponding author. Tel. : +447502548428

Email address: E.Gamba@brighton.ac.uk (E. R. Gamba)
}

Preprint submitted to Nuclear Instruments and Methods in Physics Research AMarch 7, 2019 


\section{Introduction}

The lifetimes of nuclear excited states provide essential information for the determination of reduced transition probabilities which in turn are strictly related to the nuclear wave function. Thus, nuclear lifetime measurements provide important testing for existing theoretical models.

From a few decades ago the method of measuring short delays between consecutive $\gamma$-rays, also called fast-timing technique, has been extensively used with scintillator detectors to measure nuclear lifetimes. The range of lifetimes accessible with this technique, when using $\mathrm{LaBr}_{3}(\mathrm{Ce})$ detectors, spans from a few nanoseconds to several picoseconds. [1]. Different approaches have been developed to perform fast-timing measurements, such as the Decay Slope method [2], the Convolution method and the Centroid Shift method [3-5], the Mirror Symmetric Centroid Difference (MSCD) method [6] and the Generalised Centroid Difference (GCD) method [7]. In addition, different background-subtraction procedures have been suggested [5, 6], usually applied for datasets showing peak-to-background ratios not lower than $0.4[8,9]$. Modern fast scintillator detectors like $\mathrm{LaBr}_{3}(\mathrm{Ce})$ provide an energy-dependent time resolution in the order of a hundred of picoseconds, when the aim of the fast-timing technique is often to measure lifetimes in the order of tens of picoseconds or lower. Therefore an accurate background characterisation is of crucial importance.

In this work an adaptation of a background-correction method applied for 3D $\mathrm{E}_{\gamma}-\mathrm{E}_{\gamma}-\mathrm{E}_{\gamma}$ cubes is introduced for lifetime measurements based on $\gamma-\gamma$ coincidences. The new approach is supported by a very simple but solid mathematical basis and a few experimental observations. The method has proven its effectiveness when applied to data showing very high background levels, but is, in principle, suitable to any data quality. In this work the method is applied to fast-timing measurements using the GCD method with $\operatorname{LaBr}_{3}(\mathrm{Ce})$ detectors, however nothing suggests that it restricts to a particular fast-timing method or to the type of detector used in the measurement. Furthermore the new approach is not influenced by the acquisition set-up. 
The set-up used for the examples and the measurements presented in this work, consisted of a hybrid array made of two hemispheres of 51 HPGe detectors from the Gammasphere array [10] and $25 \operatorname{LaBr}_{3}(\mathrm{Ce})$ scintillator detectors from the FATIMA collaboration [11], which were arranged in a $4 \pi$ geometry. A $34.4 \mu \mathrm{Ci}$ ${ }^{252} \mathrm{Cf}$ source was placed at the focus of the array and $\gamma$ rays emitted from the fission products were collected for 30 days. The master trigger was set to triple coincidences of the form $\mathrm{E}_{\gamma}(\mathrm{HPGe})-\mathrm{E}_{\gamma}\left(\operatorname{LaBr}_{3}(\mathrm{Ce})\right)-\mathrm{E}_{\gamma}\left(\operatorname{LaBr}_{3}(\mathrm{Ce})\right)$ and a total of $2.6 \cdot 10^{9}$ events were collected. Due to the large number of fission products, data showed relatively low statistics for the cases of interest which, combined with the high levels of background observable in fission data, makes the dataset difficult to be analysed. For fission products characterised by good fission yields, typical peak-to-background ratios are always less than 0.5 (a precise definition of this quantity is given later on in this work). For a complete description of the acquisition system see Ref. [12].

For each of the ${ }^{114} \mathrm{Pd}$ and ${ }^{110} \mathrm{Ru}$ nuclei discussed in this work, 4 Full Energy Peak (FEP) gates were applied to $\mathrm{E}_{\gamma}(\mathrm{HPGe})$ to isolate the nucleus of interest and select the excited band and 4 corresponding background gates on $\mathrm{E}_{\gamma}(\mathrm{HPGe})$ were also applied. $\mathrm{E}_{\gamma}\left(\operatorname{LaBr}_{3}(\mathrm{Ce})\right)-\mathrm{E}_{\gamma}\left(\operatorname{LaBr}_{3}(\mathrm{Ce})\right)-\Delta \mathrm{T}$ cubes were produced for each of the gates and the final $\mathrm{E}_{\gamma}\left(\operatorname{LaBr}_{3}(\mathrm{Ce})\right)-\mathrm{E}_{\gamma}\left(\operatorname{LaBr}_{3}(\mathrm{Ce})\right)-\Delta \mathrm{T}$ cube was obtained by adding together the 4 FEP-gated cubes and by subtracting the 4 associated background cubes. Gates on $\mathrm{E}_{\gamma}\left(\operatorname{LaBr}_{3}(\mathrm{Ce})\right)$ were applied in this final cube on the transitions feeding and depopulating the level of interest to perform the lifetime measurement, via the GCD method.

This work is divided into three main sections, organised as follows:

Section 2: The composition of the time background, characterising $\gamma-\gamma$ coincidence experiments, is discussed and three different background components are identified. The new procedure is introduced in terms of a correction to be applied to the position of the measured centroid of the coincidence time distribution. Essential ingredients for the correction are the "lifetimes" of the three background components.

Section 3: Two different practical approaches are suggested for evaluating the 
lifetime of the three backgrounds. The role played in the background correction by the Compton time-walk curve is discussed.

Section 4: The background correction method is then combined with the GCD method to perform lifetime measurements on ${ }^{252} \mathrm{Cf}$ fission data showing very low peak-to-background ratios. The results are shown to be consistent with the literature.

\section{Composition of the background}

In practical cases, whenever the lifetime of a nuclear excited state has to be measured, $\mathrm{E}_{\gamma}-\mathrm{E}_{\gamma}-\Delta \mathrm{T}$ cubes are often used and $\Delta \mathrm{T}$ is the measured time difference between the observation of the two $\gamma$ rays. This is defined as

$$
\Delta \mathrm{T}=\mathrm{T}\left(\mathrm{E}_{\text {stop }}\right)-\mathrm{T}\left(\mathrm{E}_{\text {start }}\right)
$$

where $\mathrm{E}_{\text {start }}$ and $\mathrm{E}_{\text {stop }}$ are the energies of the two coincident $\gamma$ rays. In the simplest case where two energy gates (slices) are imposed on the transitions feeding $\left(\mathrm{E}^{f}\right)$ and depopulating $\left(\mathrm{E}^{d}\right)$ a level of interest, the time distribution obtained from the two gates is called delayed if $\mathrm{E}^{d}$ gives the stop signal and anti-delayed if $\mathrm{E}^{d}$ is used as the start signal. To avoid confusion, in this Section $\Delta \mathrm{T}$ is defined as

$$
\Delta \mathrm{T}=\mathrm{T}\left(\mathrm{E}^{\mathrm{d}}\right)-\mathrm{T}\left(\mathrm{E}^{\mathrm{f}}\right)
$$

so that only the histogram of the delayed time distribution is produced.

Once the time distribution has been obtained, the lifetime of interest can be extracted by different approaches. For lifetimes which are long compared to the FWHM of the prompt distribution, approaches in which a function is fitted to the time distribution, or part of it, are commonly used. For example, see Ref. [13] for a recent interesting approach to find good fit limits for the decay slope method. The methods that make use of the centroid shift, e.g. the GCD method, can be applied to extract lifetimes much shorter than the prompt 
FWHM while at the same time making fewer assumptions about the shape of the distribution and the corresponding prompt response function. This work focuses on centroid-based measurements. The arguments made in this chapter about the composition of the background, and how it should be treated, can in principle be transferred to the other methods where they would be applied through corrective subtraction or an appropriate background contribution to the fit function.

In the ideal case of no background, the time distribution obtained by gating on the two $\left(\mathrm{E}^{d}\right.$ and $\left.\mathrm{E}^{f}\right)$ transitions allows a reliable measurement of the lifetime of interest. In real cases, however, Compton background from higher-energy transitions in coincidence with $\mathrm{E}^{d}$ or $\mathrm{E}^{f}$, and random coincidences modify the position of a time distribution changing the lifetime information carried by it. This effect is stronger at low energies, where the Compton components from different transitions do add up. In general the "lifetime" of the Compton background is related to the lifetimes of the decaying level producing it and, in the case of multiple Compton scattering, to the time of travel of the $\gamma$-rays detected. In order to correct the measured centroid position for the background contribution, it is thus necessary to measure the background's apparent lifetime accurately. The approach described in this work agrees with the description of the two-dimensional background discussed by Morhác̆ et al in Ref. [14], characterising multidimensional $\gamma$-ray spectra. In this work the concept is adapted to the case where the background-corrected information is a time distribution, rather than an energy spectrum.

Figure 1(a) shows the coincidence peak between the $2^{+} \rightarrow 0^{+}\left(\mathrm{E}^{d}\right.$, at $\left.241 \mathrm{keV}\right)$ and the $4^{+} \rightarrow 2^{+}\left(\mathrm{E}^{f}\right.$, at $\left.423 \mathrm{keV}\right)$ transitions in ${ }^{110} \mathrm{Ru}$ as it appears in the projection of a $\mathrm{E}_{\gamma}-\mathrm{E}_{\gamma}-\Delta \mathrm{T}$ cube. It can be observed that in the proximity of the FEP-FEP coincidence peak ( $p \mid p$ region) the background is characterised by two "ridges" at the energies of the $\mathrm{E}^{d}$ and $\mathrm{E}^{f}$ transitions $(p \mid b g$ and $b g \mid p$ regions) and by an almost flat component everywhere else (bg $\mid b g$ region). The flat component is given by coincidences between two Compton $\gamma$-rays, which are possible for any energy combination, while a coincidence between a FEP $\gamma$-ray 
and a Compton $\gamma$-ray is only possible if the FEP energy is either $\mathrm{E}^{d}$ or $\mathrm{E}^{f}$. FEP-Compton (or Compton-FEP) events are therefore the reason why the two ridges appear. The structure of the background presented in Fig. 1 is the result of the superposition of these two FEP-Compton and Compton-FEP ridges on top of the flat Compton-Compton component.

An additional component characterising the background is given by cross-talk events between adjacent detectors. For example, if a $\gamma$ ray of energy $241 \mathrm{keV}$ undergoes Compton scattering in a first detector and is then absorbed by a second one, this would appear, in the $\mathrm{E}_{\gamma}-\mathrm{E}_{\gamma}-\Delta \mathrm{T}$ cube, on a diagonal line between the points at $241 \mathrm{keV}$ on the $x$ and $y$ axes, where the exact position is given by the amount of energy released in each detector. This would happen for every photopeak energy in the spectrum, changing the appearance of the coincidence matrix considerably. In general, if these events are not rejected by active or passive shielding, or by excluding the pairs of adjacent detectors, this component should also be taken into account when estimating the background. In this work, cross-talk events were rejected by lead shields placed around the $\mathrm{LaBr}_{3}(\mathrm{Ce})$ crystal and, therefore, they are not considered in the background characterization.

It is now necessary to make a distinction between the four regions discussed so far, and shown in Fig. 1, and the time components characterising them. For instance, the $p \mid b g$ region contains both $\mathrm{E}^{d}$-Compton and Compton-Compton coincidences, which in general have distinct lifetimes. The same is true for the $b g \mid p$ region where $\mathrm{E}^{f}$-Compton and Compton-Compton coincidences are present, while in the $b g \mid b g$ region there are only Compton-Compton events. These "true" background components are indicated by the $p \mid b g^{t}$ (yellow), $b g \mid p^{t}$ (green) and $b g \mid b g^{t}$ (blue) volumes in the conceptual projection of the $\mathrm{E}_{\gamma^{-}}-\mathrm{E}_{\gamma}-\Delta \mathrm{T}$ cube in Fig. 1(b). In the same figure the "true" $\mathrm{E}^{d}-\mathrm{E}^{f}$ coincidences $\left(p \mid p^{t}\right)$ are indicated by the red volume and it is assumed that the measured $p \mid p^{m}$ time distribution, obtained by gating on the $p \mid p$ region, is the sum of all four "true" time components. The $p \mid b g^{t}$ and $b g \mid p^{t}$ components are impossible to obtain directly from the experimental data because any gate imposed on the yellow and green regions 
in Fig. 1(b) will necessarily include also the $b g \mid b g^{t}$ component. It is therefore

(a)

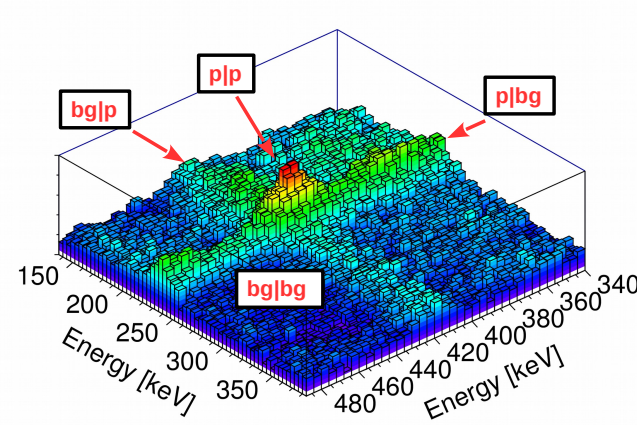

(b)

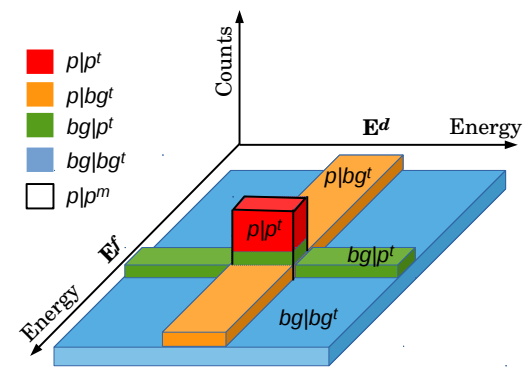

Figure 1: (a) The projection of the experimental $\gamma-\gamma-\Delta T$ cube, obtained from the ${ }^{252} \mathrm{Cf}$ source data, around the $4^{+} \rightarrow 2^{+} \rightarrow 0^{+}$cascade in ${ }^{110} \mathrm{Ru}$. The three background components are clearly visible. (b) Schematic representation of the composition of the time background inside the $E_{\gamma}-E_{\gamma}-\Delta T$ cube.

148

154

155

156

157

useful to introduce the distinction between the "true" background components considered so far, and those that can actually be measured from the three regions identified. According to the nature of the events included in each time region, the four measured time distributions $p\left|p^{m}, p\right| b g^{m}, b g \mid p^{m}$ and $b g \mid b g^{m}$, obtained by gating on the four regions, are given by

$$
p\left|b g^{m}=p\right| b g^{t}+b g \mid b g^{t},
$$

$$
b g\left|p^{m}=b g\right| p^{t}+b g \mid b g^{t},
$$

$$
b g\left|b g^{m}=b g\right| b g^{t} .
$$

${ }_{56}$ The background-subtracted time distribution given by $\mathrm{E}^{d}-\mathrm{E}^{f}$ coincidences only, can be obtained from

$$
p\left|p^{t}=p\right| p^{m}-p\left|b g^{t}-b g\right| p^{t}-b g \mid b g^{t},
$$




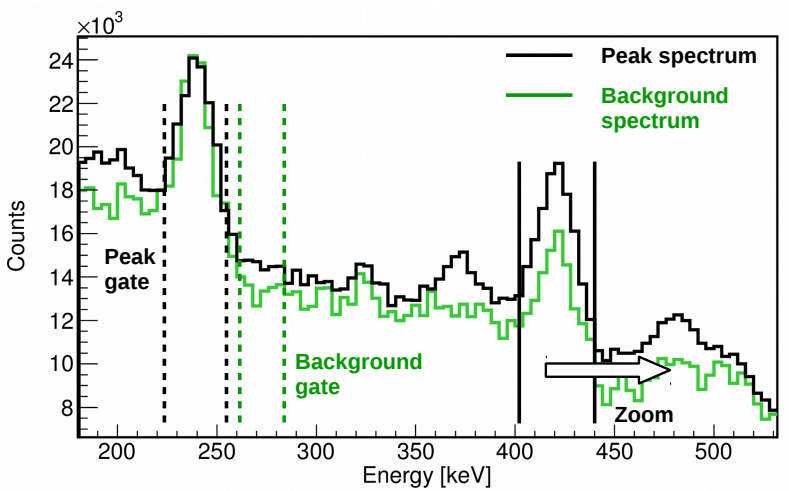

(b)

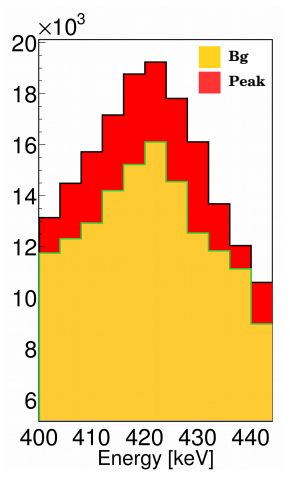

Figure 2: (a) Energy spectrum gated on the $2^{+} \rightarrow 0^{+}$transition in ${ }^{110} R u$ (black) and background energy spectrum (green) obtained by gating on the right of the $2^{+} \rightarrow 0^{+}$ transition at $241 \mathrm{keV}$ and normalised with respect to the number of counts in the $241 \mathrm{keV}$ peak. The position of the peak and background gates are indicated by the dashed black and green lines, respectively. (b) Same spectra as (a) zoomed in around the $423 \mathrm{keV}$ peak showing the background underneath the full energy peak (yellow area). The red area is related to the true number of full-energy-peak $\gamma-\gamma$ coincidences.

171 normalised with respect to the number of counts in the $241 \mathrm{keV}$ peak, and there- 
fore represents an estimate of the total background underneath the coincidence peak. It is clear from the figure that in the energy region underneath the peak at $423 \mathrm{keV}$ the background spectrum is not flat but shows a clear bump. This is nothing more than the sum of the $b g \mid p^{t}$ and $b g \mid b g^{t}$ background components, as suggested by Fig. 1(b). If the $b g \mid p^{t}$ and $p \mid b g^{t}$ components were not adding up in the $p \mid p$ region this bump would not be present. The difference between the two spectra in the $423 \mathrm{keV}$ region is therefore associated with the number of true $\mathrm{E}^{d}-\mathrm{E}^{f}$ coincidences in the $p \mid p$ region. For a similar reason in the black spectrum a bump can also be observed at $241 \mathrm{keV}$, where the gate was applied. In this case the bump results from the sum of the $p\left|b g^{t}, b g\right| p^{t}$ and $b g \mid b g^{t}$ background components. This last statement can be supported by examining the $\mathrm{E}^{d}-\mathrm{E}^{d}$ region of the $\mathrm{E}_{\gamma}-\mathrm{E}_{\gamma}-\Delta \mathrm{T}$ cube in Fig. 3 where it can be observed that a structure, resembling a coincidence peak, appears at the energies $241-241 \mathrm{keV}$. This is in fact the sum of the two ridges in the energy region around $241 \mathrm{keV}$. Only

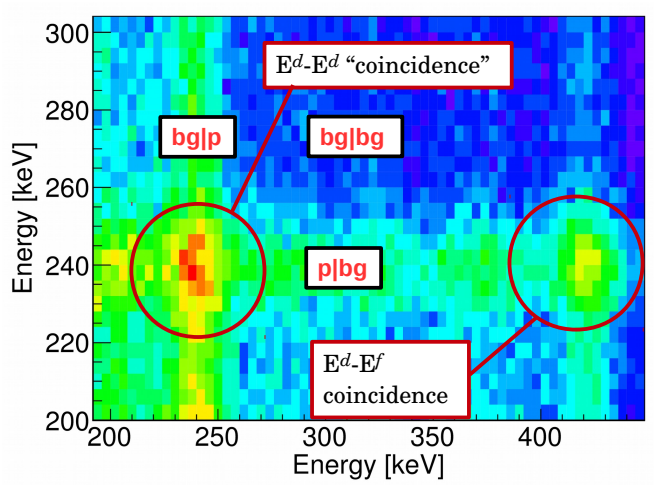

Figure 3: $E_{\gamma}-E_{\gamma}-\Delta T$ cube, showing the real coincidence peak at 241-423 keV, between the $2^{+} \rightarrow 0^{+}$and $4^{+} \rightarrow 2^{+}$transitions in ${ }^{110} R u\left(E^{d}-E^{f}\right)$ and the pure background peak at 241-241 keV $\left(E^{d}-E^{d}\right)$. The three background regions labelled in red refer to the $E^{d}-E^{d} k e V$ peak

$\gamma-\gamma$ events involving at least one Compton-scattered $\gamma$-ray are present in this peak. The three time distribution observed by gating on the three background regions around $\mathrm{E}^{d}-\mathrm{E}^{d}$, in Fig. 3, are shown in Fig. 4. It can be noticed that the measured $p \mid b g^{m}$ and $b g \mid p^{m}$ components are almost identical, as expected from the fact that the same energy transition $\mathrm{E}^{d}$ is gated twice. The "true" number 


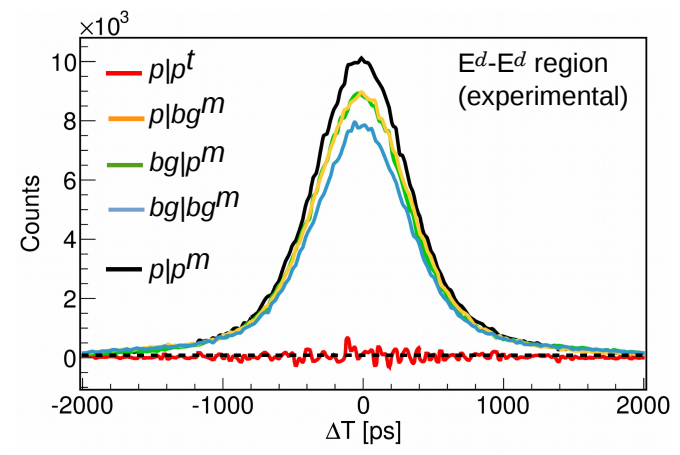

Figure 4: Measured $p \mid p^{m}$ (black), $p \mid b g^{m}$ (orange), $b g \mid p^{m}$ (green) and $b g \mid b g^{m}$ (blue) time distributions, for the $E^{d}-E^{d}$ region in Fig. 3, normalised with respect to the $p \mid p$ gate area. By applying Eq. (4) to $p \mid p^{m}$ the $p \mid p^{t}$ (red) time distribution is obtained. This is flat, as expected. See Section 3 for a discussion on the alignment of the background components.

of $\mathrm{E}^{d}-\mathrm{E}^{d}$ coincidences, expected to be zero, can be obtained by applying the sum in Eq. (4), after normalizing the three background distributions according to the $p \mid p$ gate area. Indeed, this gives the red time distribution in Fig. 4 which looks flat, confirming the composition of the background components assumed so far.

The alignment of the four time distributions shown in Fig. 4 is the result of the fact that the distributions, made of only background events, are carrying the same lifetime. In real cases, however, the centroid position of each time distribution is also affected by the energy-dependent Compton time-walk, so a correction was applied before carrying out the subtraction defined in Eq.( 4). The procedure for this will be explained in Section 3. For the rest of this Section, the centroids of the background time distributions will be assumed to be determined exclusively by the lifetime information they are carrying.

If the $\mathrm{E}^{d}-\mathrm{E}^{f}$ coincidence peak and its background components are considered instead, the "true" time distribution $p \mid p^{t}$, carrying the lifetime information, is expected to show non-zero statistics. This is illustrated in Fig. 5, where the sum of the $p \mid p^{t}$ (red), $p \mid b g^{t}$ (orange), $b g \mid p^{t}$ (green) and $b g \mid b g^{t}$ (blue) time distributions gives the measured $p \mid p^{m}$ (black) time distribution for the $\mathrm{E}^{d}-\mathrm{E}^{f}$ coincidence. In this case the $p \mid p^{t}$ time distribution is not flat and the $p \mid b g^{t}$ and $b g \mid p^{t}$ components 

procedure is necessary.

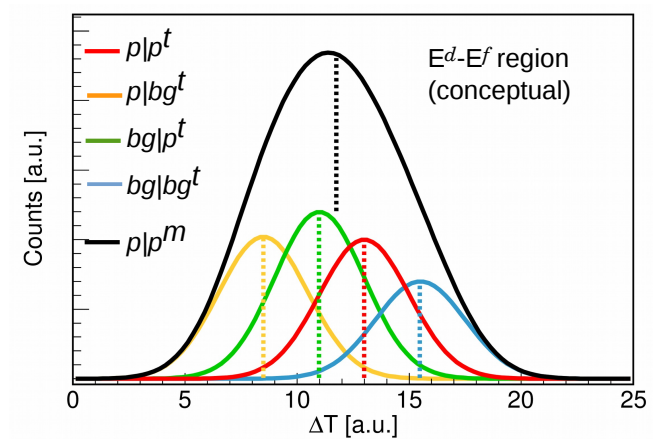

Figure 5: Time distributions of the $p \mid p^{t}$ (red), $p \mid b g^{t}$ (orange), $b g \mid p^{t}$ (green) and $b g \mid b g^{t}$ (blue) components. The sum of the four components $p \mid p^{m}$ is represented by the black time distribution. All centroids are indicated by the dashed lines.

212

are different from each other. In general the centroid positions of the individual time distributions can not be assumed to be the same. Therefore a correction

Let's now assume that the centroid position $\mathrm{C}_{p \mid p}^{m}$ has been measured from the equation for the centre of gravity

$$
\mathrm{C}=\frac{\sum_{i=a}^{b} c_{i} \cdot w_{i}}{\sum_{i=a}^{b} w_{i}}
$$

where $w_{i}$ is the content of each channel $c_{i}$ and $a$ and $b$ are the limits for the calculation of the centroid, $\mathrm{n}^{p \mid p}$ has been obtained from the number of counts of the $p \mid p^{m}$ time distribution and that the same has been done for the three background regions. The position of the "true" centroid $\mathrm{C}_{p \mid p}^{t}$ can be obtained without the need to subtract the time spectra as described by Eq. (4) but by using the following procedure on the centroid of the distribution. Let's assume that $\mathrm{C}_{p \mid p}^{m}$ is defined as the centre of gravity between the true centroid position $\mathrm{C}_{p \mid p}^{t}$ and the three background distributions with true centroid positions $\mathrm{C}_{p \mid b g}^{t}$, $\mathrm{C}_{b g \mid p}^{t}$, and $\mathrm{C}_{b g \mid b g}^{t}, \mathrm{C}_{p \mid p}^{m}$, therefore, it is given by

$$
\mathrm{C}_{p \mid p}^{m}=\frac{\mathrm{n}_{p \mid p}^{t} \mathrm{C}_{p \mid p}^{t}+\mathrm{n}_{p \mid b g}^{t} \mathrm{C}_{p \mid b g}^{t}+\mathrm{n}_{b g \mid p}^{t} \mathrm{C}_{b g \mid p}^{t}+\mathrm{n}_{b g \mid b g}^{t} \mathrm{C}_{b g \mid b g}^{t}}{\mathrm{n}_{p \mid p}^{t}+\mathrm{n}_{p \mid b g}^{t}+\mathrm{n}_{b g \mid p}^{t}+\mathrm{n}_{b g \mid b g}^{t}}
$$


${ }_{224}$ where the "true" number of counts $\mathrm{n}_{p \mid p}^{t}, \mathrm{n}_{p \mid b g}^{t}, \mathrm{n}_{b g \mid p}^{t}$ and $\mathrm{n}_{b g \mid b g}^{t}$ are those of the four time distributions in Fig. 5. From Eq. (6) the analytical expression of the position $\mathrm{C}_{p \mid p}^{t}$ can easily be obtained. Since only "true" Compton-Compton events are present in the blue region of Fig. 1(b), it is straightforward to assume that

$$
n_{b g \mid b g}^{t}=n_{b g \mid b g}^{m}, \quad \text { and } \quad \mathrm{C}_{b g \mid b g}^{t}=\mathrm{C}_{b g \mid b g}^{m}
$$

229 On the other hand, by gating on the $p \mid b g$ region, what is obtained is a sum 230 of the $p \mid b g^{t}$ and $b g \mid b g^{t}$ components, with number of counts $\mathrm{n}_{p \mid b g}^{m}$ and centroid ${ }_{231}$ position $\mathrm{C}_{p \mid b g}^{m}$, and therefore

$$
\mathrm{n}_{p \mid b g}^{m}=\mathrm{n}_{p \mid b g}^{t}+\mathrm{n}_{b g \mid b g}^{t},
$$

232 and

$$
\mathrm{C}_{p \mid b g}^{m}=\frac{\mathrm{n}_{p \mid b g}^{t} \mathrm{C}_{p \mid b g}^{t}+\mathrm{n}_{b g \mid b g}^{t} \mathrm{C}_{b g \mid b g}^{t}}{\mathrm{n}_{p \mid b g}^{t}+\mathrm{n}_{b g \mid b g}^{t}} .
$$

${ }_{233}$ From Eqs. 8 and 9 the true number of counts and centroid for the $p \mid b g$ back234 ground components are given by

$$
\mathrm{n}_{p \mid b g}^{t}=\mathrm{n}_{p \mid b g}^{m}-\mathrm{n}_{b g \mid b g}^{m},
$$

235 and

$$
\mathrm{C}_{p \mid b g}^{t}=\frac{\mathrm{n}_{p \mid b g}^{m} \mathrm{C}_{p \mid b g}^{m}-\mathrm{n}_{b g \mid b g}^{m} \mathrm{C}_{b g \mid b g}^{m}}{\mathrm{n}_{p \mid b g}^{t}}
$$

${ }_{236}$ Similarly, the true number of counts and centroid position for the $b g \mid p$ back237 ground are given by

$$
\mathrm{n}_{b g \mid p}^{t}=\mathrm{n}_{b g \mid p}^{m}-\mathrm{n}_{b g \mid b g}^{m},
$$

238 and

$$
\mathrm{C}_{b g \mid p}^{t}=\frac{\mathrm{n}_{b g \mid p}^{m} \mathrm{C}_{b g \mid p}^{m}-\mathrm{n}_{b g \mid b g}^{m} \mathrm{C}_{b g \mid b g}^{m}}{\mathrm{n}_{b g \mid p}^{t}} .
$$


${ }_{239}$ Finally, following the same logic of Eq. (4), $\mathrm{n}_{p \mid p}^{t}$ is given by

$$
\mathrm{n}_{p \mid p}^{t}=\mathrm{n}_{p \mid p}^{m}-\mathrm{n}_{p \mid b g}^{m}-\mathrm{n}_{b g \mid p}^{m}+\mathrm{n}_{b g \mid b g}^{m}
$$

240 By substituting Eqs. (8) - (14) into Eq. (6) the centroid position of the $p \mid p^{t}$ time 241 distribution, is

$$
\mathrm{C}_{p \mid p}^{t}=\frac{\mathrm{n}_{p \mid p}^{m} \mathrm{C}_{p \mid p}^{m}-\mathrm{n}_{p \mid b g}^{m} \mathrm{C}_{p \mid b g}^{m}-\mathrm{n}_{b g \mid p}^{m} \mathrm{C}_{b g \mid p}^{m}+\mathrm{n}_{b g \mid b g}^{m} \mathrm{C}_{b g \mid b g}^{m}}{\mathrm{n}_{p \mid p}^{m}-\mathrm{n}_{p \mid b g}^{m}-\mathrm{n}_{b g \mid p}^{m}+\mathrm{n}_{b g \mid b g}^{m}}
$$

242 Equation (15) therefore represents the background corrected position of the $\mathrm{E}^{d}-\mathrm{E}^{f}$ time distribution. This can also be written in a more familiar form as

$$
\mathrm{C}_{p \mid p}^{t}=\frac{\mathrm{C}_{p \mid p}^{m}}{\Pi}-\frac{\mathrm{C}_{p \mid b g}^{m}}{\Pi_{p \mid b g}}-\frac{\mathrm{C}_{b g \mid p}^{m}}{\Pi_{b g \mid p}}+\frac{\mathrm{C}_{b g \mid b g}^{m}}{\Pi_{b g \mid b g}}
$$

where $\Pi$ is the peak-to-total-background ratio defined as

$$
\Pi=\frac{\mathrm{n}_{p \mid p}^{m}-\mathrm{n}_{p \mid b g}^{m}-\mathrm{n}_{b g \mid p}^{m}+\mathrm{n}_{b g \mid b g}^{m}}{\mathrm{n}_{p \mid p}^{m}}
$$

and the $\Pi_{i}$ terms are peak-to-partial-background ratios, calculated with respect to each background component.

The background correction approach suggested by Eq. (15) or (16) is usually preferable to a physical subtraction between time spectra as the one in Eq. (4), for at least three reasons:

- The energy-dependant time resolution of a fast-timing system, implies that the three background time distributions to be subtracted from $p \mid p^{m}$ have FWHM values which are different from the $p \mid p^{m}$ one and also from each other. The difference in FWHM is negligible when the background gates are close in energy to the $p \mid p$ one, but will increase as the difference between the peak and background gates increases. This could lead to a situation where, by using Eq. (4), the FWHM of the final time distribution might be distorted by the subtraction. 
- Whenever the data is characterised by low peak-to-background ratios, the approach of Eq. (4) can produce a $p \mid p^{t}$ time distribution, showing low statistics and large error bars on its data points.

- The four time distributions are characterised by different Compton timewalks. This requires the time distributions of the three background components to be shifted or the three background centroid values in Eq. 15 to be modified according to it. The Compton time-walk correction works better in the second case, as explained in Section 3.

As a final remark it is important to discuss the role played by the background given by random coincidences that has so far been ignored. This is because it can be assumed that random coincidences produce a similar structure to that given by the three Compton components. The number of FEP-random coincidences will be larger than the number of Compton-random coincidences, which in turn is expected to be larger than the number of random-random coincidences, these last two assumed to occur for any energy combination, similarly to the ComptonCompton component. This ensures that when Eq. (15) or (16) are applied, the right proportion of random background is also taken into account.

\section{Two approaches for determining the lifetime of the background}

In general the time distribution $p \mid p^{m}$ is obtained by gating on both $\mathrm{E}^{d}$ and $\mathrm{E}^{f}$ transitions, feeding and depopulating the level of interest. The number of counts represented by $\mathrm{n}_{p \mid p}^{m}$ is measured by integration of the time distribution and the centroid $\mathrm{C}_{p \mid p}^{m}$ is obtained from Eq. 5. The experimental challenge is to obtain the centroid positions and number of counts (or $\Pi$ and $\Pi_{i}$ ratios) for the three background components such that they truly reflect the background contaminating the $p \mid p^{m}$ time distribution, so that they can be used in Eq. (15) (or Eq. (16)). There are different ways to do so: two different approaches, based on slightly different assumptions, are suggested in this work. The measurement of the three background components is presented here for the case of 
the delayed time distribution, but in all systems where both delayed and antidelayed branches are used (as for the GCD method), the procedure has to be applied to both of them independently and, therefore, a total of six background components have to be determined.

\subsection{Interpolation}

Let's consider, for example, the $b g \mid p$ region of the $4^{+} \rightarrow 2^{+} \rightarrow 0^{+}$cascade in ${ }^{110} \mathrm{Ru}$. The behaviour of this background component, in energy and time, can be observed by applying a first gate on the $\mathrm{E}^{f}(423 \mathrm{keV})$ transition. The energy spectrum in Fig. 6(a), zoomed around the $\mathrm{E}^{d}(241 \mathrm{keV})$ transition, is the result of such a gate. The $b g \mid p^{m}$ background time component is estimated by applying the second gate in the energy regions around the $\mathrm{E}^{d}$ energy peak as indicated by the black arrows. The gate width has to be kept constant to give the same weight to each measurement. For each gate one has to make sure that no contaminant peaks are included so that the $b g \mid p^{m}$ component is really the only element contributing.

In Fig. 6(b) the centroid position of the obtained time distributions (one for each gate) is plotted versus the centroid of the second gate. The set of points representing the centroids of the time distribution (black dots) are then interpolated (red solid line) to estimate the value of $p \mid b g^{m}$ at the energy of $\mathrm{E}^{d}$ (green marker). Three different functions have been used to perform the fit: a first order polynomial, a second order polynomial and a curve with equation

$$
\mathrm{C}_{b g \mid p}\left(E_{\gamma}\right)=\frac{a}{\sqrt{E_{\gamma}+b}}+c E_{\gamma}+d
$$

where $a, b, c$ and $d$ are the fit parameters. This is an empirical formula used to calibrate the FEP time-walk when using the GCD or MSCD methods [15] and it was constructed by adding a linear term to the equation describing the Constant Fraction Discriminator (CFD) time-walk. The FEP time walk-curve obtained in the GCD method from Eq. 18 is called Prompt Response Difference (PRD) curve and it will be used later on in this work. 
Provided that there are enough points to produce a reliable fit, Eq. 18 is also useful to describe the timing background and it is the one that gives the best $\tilde{\chi}^{2}$ value. If only a few background gates are applied, especially if they lie close to the coincidence peak, a second-order-polynomial fit is a reasonable approximation. This procedure is repeated for each of the three background

(a)

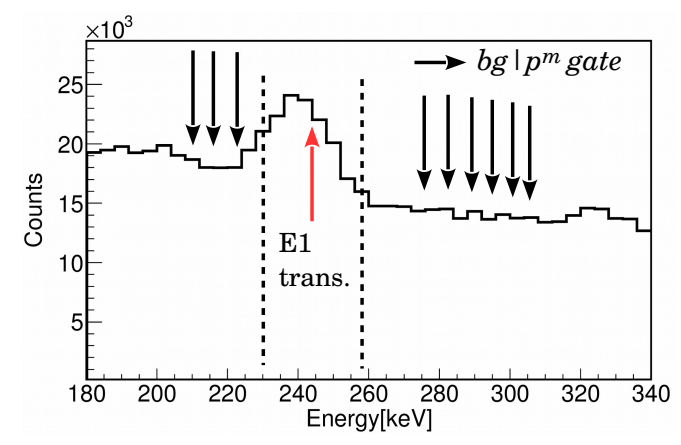

(b)

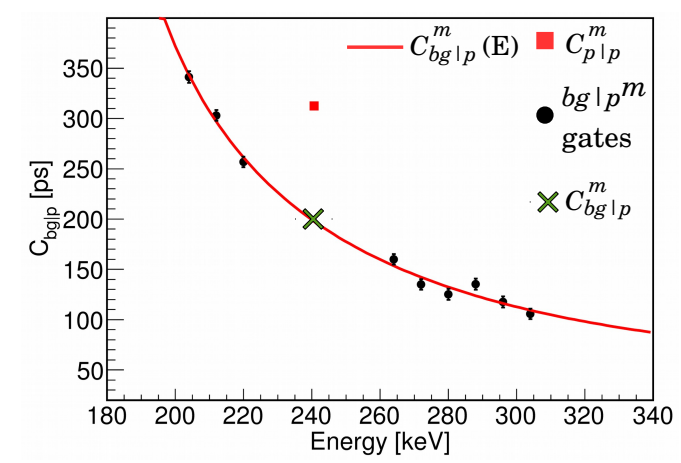

Figure 6: (a) $\gamma$-ray spectrum gated on the $4_{1}^{+} \rightarrow 2_{1}^{+}$transition in ${ }^{110} R u$. The black arrows indicate the $b g \mid p^{\text {gates }}$, while the red arrow indicates the $2_{1}^{+} \rightarrow 0_{g . s .}^{+}$transition for which the $p \mid p^{m}$ time distribution is obtained. (b) Interpolation of the centroid positions for the different $b g \mid p^{m}$ gates (black points). The bg $\mid p^{m}$ value to be used in Eq. (15) is given by the green marker. The measured centroid position for $p \mid p^{m}$ is the red square.

317

regions as shown in Fig. 7, and the resulting background centroid values are used in Eq. (15). When using this approach $\mathrm{n}_{p \mid p}^{m}$ is the number of counts of the $p \mid p^{m}$ distribution, while for each of the three background components the energy regions used to extract $\mathrm{n}_{p \mid b g}^{m}, \mathrm{n}_{b g \mid p}^{m}$ and $\mathrm{n}_{b g \mid b g}^{m}$ have to be consistent with those 
used to obtain the data points, but they also need to be as close as possible to the coincidence peak. If, for example, $b g \mid p^{m}$ background gates were taken both to the left and to the right of the coincidence peak, then both sides have to be included in the evaluation of $\mathrm{n}_{b g \mid p}^{m}$. It is important to stress that, because of Eq. (14), the measured number of counts for the background components will necessary affect also $\mathrm{n}_{p \mid p}^{t}$, therefore, extra care is necessary when choosing the background regions.

Some discussion is necessary for the inclusion of points to the left of the $\mathrm{E}^{d}$ energy peak. These are affected by the Compton background produced by the transition itself. Whether to include them or not, in this or any form of background correction, is a decision that depends on several factors, such as the energy resolution of the system, the energy at which the peak lies with respect to the Compton edges of the other energy transitions, and the number of background samples available for the interpolation. For a system where the energy resolution is poor, the $\mathrm{E}^{d}$ peak could be significantly and non-uniformly affected by its own Compton background. By gating to the left of the $\mathrm{E}^{d}$ peak this effect is partially taken into account. If the $\mathrm{E}^{d}$ peak lies at high energies the background to its right (above $1 \mathrm{MeV}$ in this dataset) can be negligible and therefore only the left component should be considered. The position of the Compton edge for each peak in the energy spectrum should also be calculated in order to determine whether the left and right background regions are expected to be different or can be assumed to carry similar lifetime information. Finally, if the number of contaminant peaks around $\mathrm{E}^{d}$ is quite large it can be difficult to obtain a good number of points to perform the fit and including the region to the left of $\mathrm{E}^{d}$ is a reasonable option. In general, in order to have a better estimate of each background component, a case-by-case evaluation is recommended.

\subsection{The three samples}

The three samples approach is used when the large number of contaminant peaks does not allow to select enough gates for the interpolation method and each background component can be estimated by only one energy gate (or two 


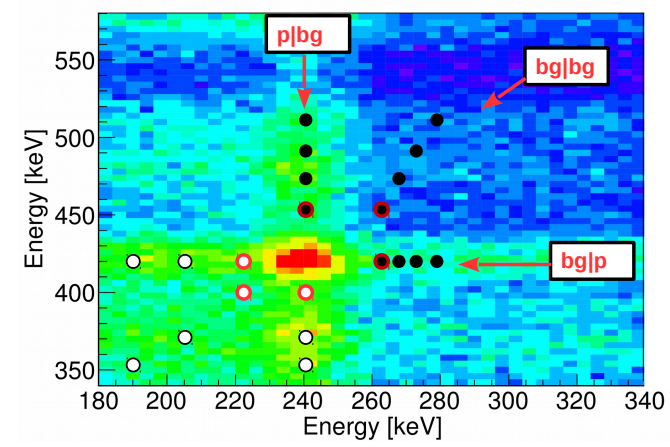

Figure 7: Projection of the $E_{\gamma}-E_{\gamma}-\Delta T$ cube showing some hypothetical gates to be applied in the three background regions to the right of the coincidence peak (black dots) and to its left (white dots).

if the left-side is also considered) taken as close as possible to the coincidence peak. For instance the regions indicated by the three dots encircled in red in Fig. 7 could be gated to obtain three background time distributions whose number of counts and centroids are used in Eq. (15). The centroid of each background time distribution must be representative only of the background's lifetime, however in real cases it is also shifted by the Compton time-walk. This is similar to the FEP-FEP time-walk but characterising only FEP-Compton events, and it is energy dependent. Therefore, the centroid of each background time distribution must be corrected according to the Compton time-walk, before it can be used in Eq. (15).

The value of this correction is obtained from the Compton curve [1]. After this correction has been applied the only component affecting the position of the centroids is the lifetime information that each time distribution carries. In the interpolation method this effect is already taken into account by the fitted curves obtained to estimate the background components and therefore no Compton time-walk correction is necessary in this case.

\subsubsection{The Compton curve}

The Compton time-walk curve is constructed in the same way as the FEP time-walk curve except that the Compton background is instead used as a start or stop signal. The curve will in general look different from the FEP curve 
because, for a given energy, the time response of photoelectric absorption and the Compton effect is different $[16,17]$. It is logical to assume that the origin of the difference between the Compton time-walk and the full-energy-peak timewalk is given by particular events that contribute only to the background and never to the full-energy peaks. According to Ref. [16] this difference is usually given by spurious events like $\gamma$ rays scattering from the detector's shield to the inside of the crystal. In the set-up described in Section 1 lead shields were used between adjacent $\operatorname{LaBr}_{3}(\mathrm{Ce})$ detectors to prevent cross-talk events but, in general, multiple Compton scattering events between different detectors might also contribute to the Compton time-walk. On the other hand, a $\gamma$ ray scattering one or multiple times inside the same detector might result in a Compton event but, if it finally undergoes photoelectric absorption, it might also appear at the photopeak energy. Therefore, this type of event shouldn't contribute to the difference between the two time-walk curves.

To construct the Compton curve a source of ${ }^{88} \mathrm{Y}$ ( which decays via electron capture into ${ }^{88} \mathrm{Sr}$ ) was placed at the focus of the $\mathrm{LaBr}_{3}(\mathrm{Ce})$ array. Figure $8(\mathrm{a})$ shows the $\gamma$-ray spectrum obtained from this source. This choice was made for two reasons. The first is that the lifetime of the $2_{1}^{+}$state in ${ }^{88} \mathrm{Sr}$ is negligible with respect to the time resolution of the system and therefore the only component affecting the position of the centroids is due to the Compton time-walk. The second reason is the absence of other strong contaminant transitions, which makes the energy spectrum and the time information very clean. After gating on the $2_{1}^{+} \rightarrow 0_{g . s .}^{+}$transition in ${ }^{88} \mathrm{Sr}$, what is obtained is a textbook $\gamma$-ray spectrum of Compton background as shown in Fig. 8(b). Note that in Fig. 8(b) the residual background component is two orders of magnitude lower than the gated spectrum, and therefore it is negligible.

In order to construct a Compton curve to be used with the GCD method, $\gamma-\gamma$ coincidences from the ${ }^{88} \mathrm{Y}$ source data were used to create a $\gamma-\gamma-\Delta \mathrm{T}$ cube, where the information from the detector with the smaller ID was put on the $x$ axis and the other on the $y$ axis. This avoids the cube from being filled twice with the same pair of $\gamma$ rays and also makes it asymmetrical. A slightly 
(a)

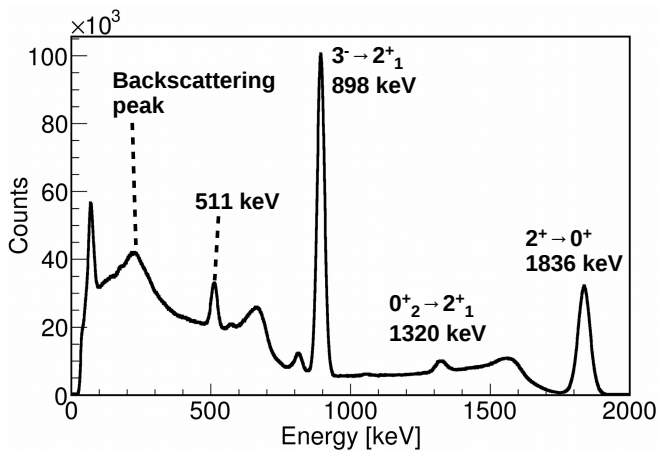

(b)

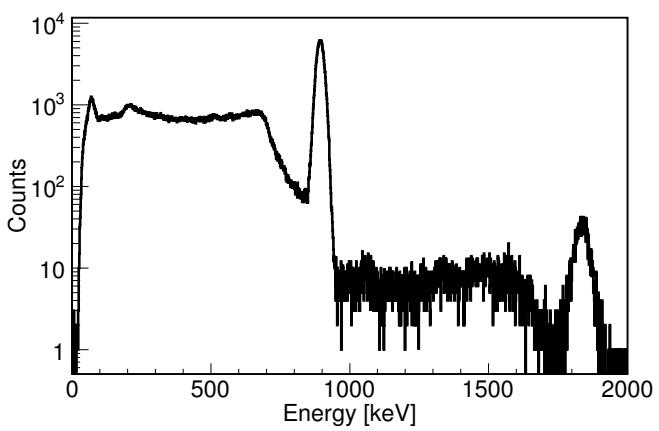

Figure 8: (a) $\mathrm{LaBr}_{3}(\mathrm{Ce}) \gamma$-ray spectrum obtained from a ${ }^{88} \mathrm{Y}$ source. The $2_{1}^{+} \rightarrow 0_{\text {g.s. }}^{+}$ $(1836 \mathrm{keV}), 3_{1}^{-} \rightarrow 2_{1}^{+}(898 \mathrm{keV})$ and $0_{2}^{+} \rightarrow 2_{1}^{+}(1320 \mathrm{keV})$ transitions are visible, together with the $511 \mathrm{keV}$ peak and the backscattering peak at $240 \mathrm{keV}$. (b) $\mathrm{LaBr}_{3}(\mathrm{Ce})$ $\gamma$-ray spectrum gated on the $2_{1}^{+} \rightarrow 0_{g . s .}^{+}$. transition in ${ }^{88} \mathrm{Sr}$. In the region below $898 \mathrm{keV}$ the spectrum is showing a very clean Compton distribution. The time contribution of the small number of counts of the residual Compton events from the 1836 peak (the region above $898 \mathrm{keV}$ ) is negligible. The spectrum is not background subtracted.

different approach for time-walk calibrations was suggested in Ref. [18], where the advantages of using symmetric cubes are shown. The Compton curve able to describe the delayed component of the background time-walk information, can be obtained from the centroid positions of the time distributions obtained by gating on a FEP region as a start and on several narrow background regions as the stop. The position of the FEP gate is fixed and by allowing the energy of the background gate to span the Compton continuum produced by the other peaks, it is possible to measure different time-walk values in the energy region. 
The FEP gate is than moved to another peak and the procedure is repeated. The anti-delayed Compton curve is obtained in the same way but using the FEP gate as the stop and the background gates as the start. For the GCD method a single Compton curve is used (see later in this Section) and this can in principle be obtained from the difference between these two time-walk curves. However, in practice, each point of the Compton curve is the centroid difference, $\Delta \mathrm{C}$, between the delayed and anti-delayed time distributions obtained by gating on a FEP region as a start, and a narrow background region as a stop respectively (delayed), and vice versa (anti-delayed). Equation 18 is then used to fit the data points.

For the Compton curve presented in this work the data points were obtained by gating on the $2_{1}^{+} \rightarrow 0_{g . s .}^{+}(1836 \mathrm{keV})$ transition in ${ }^{88} \mathrm{Sr}$ and $\Delta \mathrm{C}$ was measured for several regions between $\sim 100$ and $\sim 200 \mathrm{keV}$ and in the region $[\sim 300, \sim 650]$ (in order to avoid the backscattering peak at $\sim 240 \mathrm{keV}$ ). This gave a first set of 36 points. The same was done for the $3_{1}^{-} \rightarrow 2_{1}^{+}$transition at $898 \mathrm{keV}$ and the two background regions $[\sim 950, \sim 1200] \mathrm{keV}$ and $[\sim 1400, \sim 1550] \mathrm{keV}$, obtaining a second set of 36 points. The gap between these two last regions is where the $1320 \mathrm{keV}$ peak lies. This second set of points was then shifted to be consistent with the choice of a reference energy at $1836 \mathrm{keV}$ (the same procedure is usually applied to build the PRD curve). The new set of 72 points was then fitted using Eq. (18), to obtain the curve shown in Fig. 9(a). At the reference energy of $1836 \mathrm{keV}$ the Compton curve should have a value of zero ps. A value of $-5 \mathrm{ps}$ is obtained at $1836 \mathrm{keV}$ but this is still a good approximation considering that it was not possible to obtain reliable points in the region of energy above $1600 \mathrm{keV}$. The points on Fig. 9(b) shows the residuals of the fit, and the blue lines indicate the coincidence interval of $1 \sigma$.

The PRD curve is obtained following a very similar procedure to the one used for the Compton curve, explained in Ref.[6], with the difference that both start and stop gates are set on FEP regions only. Figure 10 shows a comparison between the PRD curve, obtained with the same set-up, and the Compton curve presented in this Section. Both curves were shifted in order to be represented 
with respect to the reference energy of $344.3 \mathrm{keV}$, from the $2_{1}^{+} \rightarrow 0_{g . s .}^{+}$transition in ${ }^{152} \mathrm{Gd}$. The two curves are observed to cross at this energy value, that corresponds to the ideal case of true prompt centroids where the energies of the start and stop transitions are identical, from which $\Delta \mathrm{C}=0 \mathrm{ps}$. The figure shows that, for energy values different from $344.3 \mathrm{keV}$, the two time-walk curves have different energy dependences, as expected. Before showing, in practice, how to

(a)

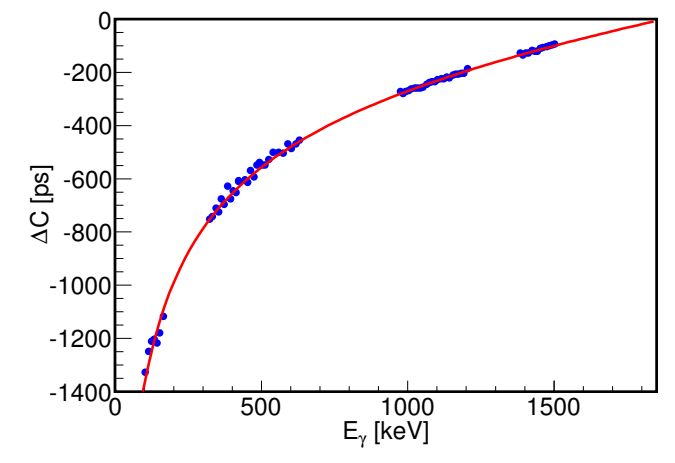

(b)

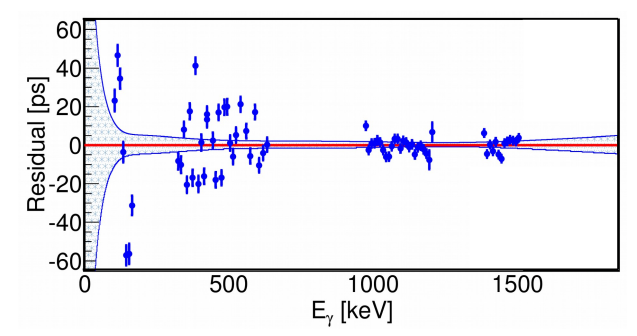

Figure 9: (a) The Compton curve (red) parametrized with: $a=-17800(300)$, $b=35(3), c=0.143(3)$ and $d=138(13)$ using the formula in Eq. (18), together with the 72 points (blue) used for the fit. The distribution of the points is related to the four energy regions considered (see text). (b) The blue lines represent a confidence interval of $1 \sigma$ of the fit in (a).

apply the Compton time-walk correction to the background time distributions, it is necessary to discuss about how absolute the Compton curve, as it is described in this Section, can be. It is reasonable to assume that the Compton curve is set-up-dependent but whether there is only one Compton curve to be obtained from a particular experimental set-up (as for the PRD curve), or not, is an important question that needs to be answered. In the fundamental work by 


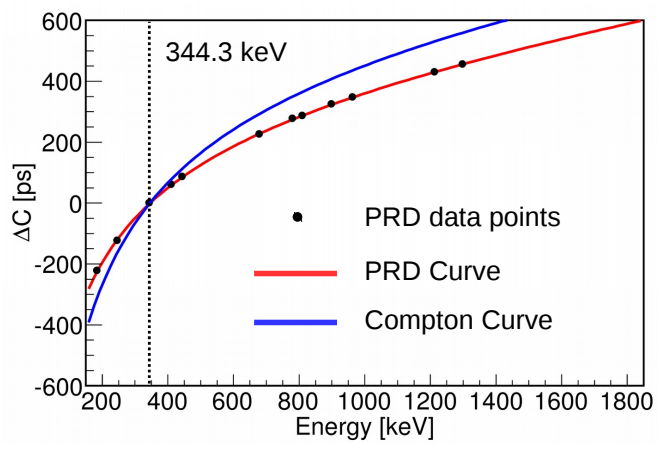

Figure 10: Comparison between the PRD curve (red) and the Compton Curve (blue), both are plotted using $344.3 \mathrm{keV}$, from the $2_{1}^{+} \rightarrow 0_{\text {g.s. }}^{+}$transition in ${ }^{152} \mathrm{Gd}$, as the reference energy. Data points for the PRD curve are represented by the black dots, the size of the error bars is comparable to that of the dots.

Mach et al. [1] the equivalent of a Compton curve is obtained from $\beta-\gamma(\mathrm{HPGe})-$ $\gamma\left(\mathrm{BaF}_{2}\right)$ coincidences, but only for the delayed time component. Two different curves are obtained using a ${ }^{24} \mathrm{Na}$ source, one from the Compton background produced by the $4_{1}^{+} \rightarrow 2_{1}^{+}$transition in ${ }^{24} \mathrm{Mg}$ at $2.75 \mathrm{MeV}$ and a second one from the $2_{1}^{+} \rightarrow 0_{g . s .}^{+}$transition at $1.37 \mathrm{MeV}$. In Fig. 3 of the paper [1], these are shown to have similar shapes, but to be shifted by a certain number of picoseconds. According to the authors this difference is given by two factors: the different lifetimes of the transitions producing the Compton background, and the different average scattering-angle of the $\gamma$ rays inside the detector, which results in a different delay. In this sense, the shift applied between the two sets of 36 points (from the $3_{1}^{-} \rightarrow 2_{1}^{+}$and $2_{1}^{+} \rightarrow 0_{1}^{+}$transitions in ${ }^{88} \mathrm{Sr}$ ), when building the Compton curve, is a correction to this difference. Therefore, the Compton curve can be assumed to be independent of the energy of the transitions producing the Compton background. Since the Compton time-walk correction is obtained from the Compton curve by the difference between the time-walk at the energies of the coincidence peak and the background gates, then the absolute value of the correction is only shape-dependent. A reference value, given by

$$
\operatorname{Compt}_{p \mid p}=\operatorname{Compt}\left(\mathrm{E}^{\mathrm{f}}\right)-\operatorname{Compt}\left(\mathrm{E}^{\mathrm{d}}\right),
$$


is equivalent to the "zero" position of the real background underneath the coincidence peak. If, for example, the $p \mid b g^{m}$ component has been obtained from two gates at the energies $\mathrm{E}^{d}$ and $\left(\mathrm{E}^{f}+x\right)$, then

$$
\operatorname{Compt}_{p \mid b g}=\operatorname{Compt}\left(\mathrm{E}^{\mathrm{f}}+x\right)-\operatorname{Compt}\left(\mathrm{E}^{\mathrm{d}}\right) .
$$

The shift to be applied to the $p \mid b g^{m}$ time distribution is equal to

$$
S_{p \mid b g} \simeq \frac{\left(\text { Compt }_{p \mid b g}-\text { Compt }_{p \mid p}\right)}{2} .
$$

When using the GCD method, the sign of the correction given in Eq. 21 has to be opposite for the delayed and anti-delayed components. In general the two time distributions are not exactly symmetric with respect to the $\Delta \mathrm{T}=0$ axis, therefore, it can be assumed that the two prompt Compton curves used to build the Compton curve in Fig. 9 are not symmetric either. In this case dividing by a factor of two in Eq. 21 is an approximation; the two prompt Compton curves should be kept separate and individual corrections should be calculated for the delayed and anti-delayed $p \mid b g^{m}$ components. However the approximation is a reasonable one as shown by the comparison between Fig. 11 (before the correction) and Fig. 12 (after). The effect of the Compton correction is a natural alignment of the three background components with respect to $p \mid p^{m}$. Since the lifetime of the three background components are now the only element defining the position of each of the three centroids it is not expected that the four distributions would be perfectly aligned after the correction. The shift between the background time distributions shown in Fig. 11 and those shown in Fig. 12 is useful to visualise the effect of the Compton correction, but in practice it is not recommended to apply a physical shift to the time distributions. The shift given in Eq. 21 must be necessarily converted (and therefore rounded) into number of channels and depending on how many ps-per-channel are used, this can produce an even further approximation. On the other hand if the shifts calculated are used to modify the measured centroid position of each background component 

$\mathrm{C}_{p \mid b g}^{m}, \mathrm{C}_{b g \mid p}^{m}$ and $\mathrm{C}_{b g \mid b g}^{m}$, which are then used in Eq. 15, this problem is overcome.

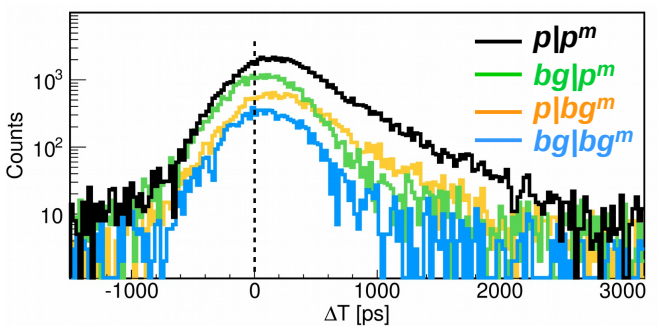

Figure 11: The three measured background time distributions $p\left|b g^{m}, b g\right| p^{m}$ and $b g \mid b g^{m}$ (green, yellow and orange, respectively) without Compton time-walk correction and the $p \mid p^{m}$ time distribution (black). The dashed line is at $\Delta T=0$.

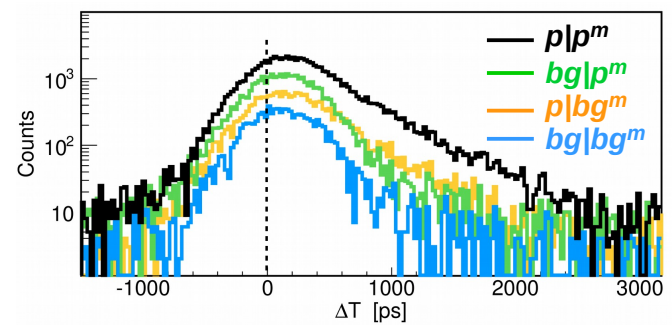

Figure 12: Same as Fig. 11, after applying the Compton correction to each of the three background time distributions.

\section{Measurement examples}

The measurements presented here were performed with the set-up described in Section 1. For each lifetime measurement both delayed and anti-delayed time distributions are produced. These are made of different $\gamma-\gamma$ coincidences but carry the same lifetime information. Therefore the background correction procedure has to be applied on both of them. The Compton curve in Fig. 9 and a PRD curve with parameters: $\mathrm{a}=-13070(3602) \mathrm{b}=45(59) \mathrm{c}=0.1609(500)$ $\mathrm{d}=603(150)$ from Eq. 18 were obtained for this set-up.

\subsection{The $2^{+}$state in ${ }^{110} R u$}

The lifetime of the $2_{1}^{+}$state in ${ }^{110} \mathrm{Ru}$ has been measured using the background correction approaches described in both Sections 3.1 and 3.2 . ${ }^{110} \mathrm{Ru}$ is 
among the nuclei showing the best fission yields for ${ }^{252} \mathrm{Cf}$ (3.6 nuclei produced in 100 fission events), and it therefore provides reasonable statistics. In order to isolate the $2_{1}^{+}$state in ${ }^{110} \mathrm{Ru}$ four different energy gates were imposed on the Gammasphere array, on the ground-state-band $12_{1}^{+} \rightarrow 10_{1}^{+}(888 \mathrm{keV}), 10_{1}^{+} \rightarrow 8_{1}^{+}$ $(815 \mathrm{keV}), 8_{1}^{+} \rightarrow 6_{1}^{+}(705 \mathrm{keV})$ and $6_{1}^{+} \rightarrow 4_{1}^{+}(576 \mathrm{keV})$ transitions. For each FEP energy gate a background gate was selected and the procedure described in Section 1 was applied in order to obtain the total background subtracted $\mathrm{E}_{\gamma}-\mathrm{E}_{\gamma}-\Delta \mathrm{T}$ cube.

The resulting HPGe and $\mathrm{LaBr}_{3}$ energy spectra (the $\mathrm{LaBr}_{3}(\mathrm{Ce})$ spectrum is the projection of the final cube) are presented in Fig. 13(a), where transitions belonging to ${ }^{110} \mathrm{Ru}$ and its fission partners ${ }^{136,138} \mathrm{Xe}$ are labelled. The procedure to obtain a time distribution for the $2^{+}$level in ${ }^{110} \mathrm{Ru}$ begins from the matrix in Fig. 13(b). This is actually the two-dimensional projection of a start and stop $\mathrm{E}_{\gamma}\left(\operatorname{LaBr}_{3}(\mathrm{Ce})\right)-\mathrm{E}_{\gamma}\left(\operatorname{LaBr}_{3}(\mathrm{Ce})\right)-\Delta \mathrm{T}$ cube, where

$$
\Delta T=T_{E y}-T_{E x}
$$

Consistently with the procedure used to make the PRD and Compton curves, also for the fission data the information from the detector with the smaller ID was put on the $x$ axis and the one with the larger ID on the $y$ axis. Both the delayed (bottom-right) and anti-delayed (top-left) coincidence peaks can be observed in the $\gamma-\gamma$ matrix presented in Fig. $13(\mathrm{~b})$, for the $2_{1}^{+} \rightarrow 0_{g . s .}^{+}(241 \mathrm{keV})$ and $4_{1}^{+} \rightarrow 2_{1}^{+}(423 \mathrm{keV})$ transitions in ${ }^{110} \mathrm{Ru}$. As expected they are different from each other, but they are also expected to carry same lifetime information. By comparing the two energy spectra in Fig. 13(a) background regions free of contaminant peaks, to be used for the background correction, were identified. These are indicated in the $\mathrm{E}_{\gamma}-\mathrm{E}_{\gamma}$ matrix. The position of the Compton-edge for each $\mathrm{LaBr}_{3}(\mathrm{Ce})$ contaminant peak in Fig. 13(a) was calculated and, since none of them lies to the immediate left of the coincidence peak, the decision to apply background gates also to the left side was taken. These gates were selected as close as possible to the peaks in order to minimize the contributions from the 
(a)

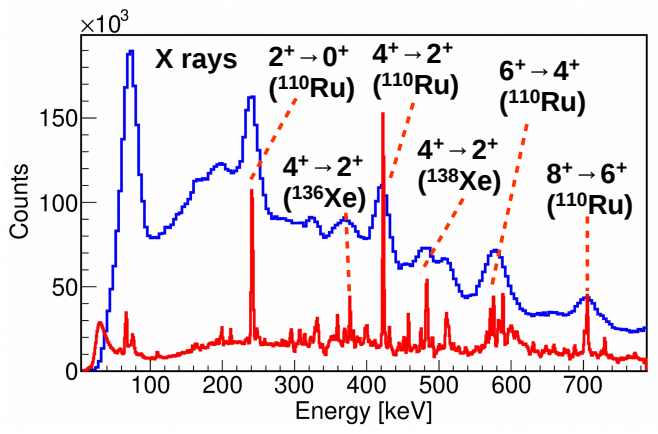

(b)

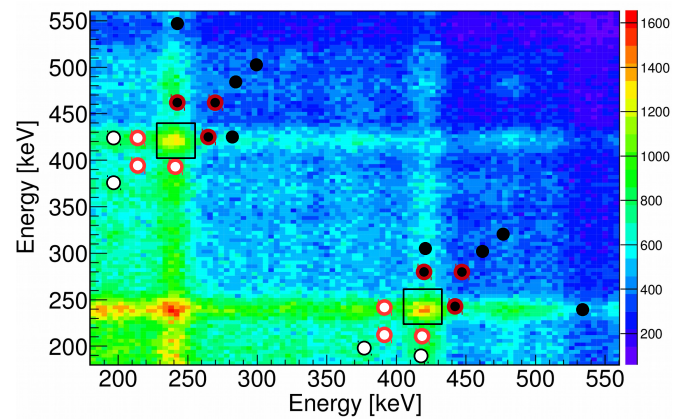

Figure 13: (a) Sum HPGe (red) and $\mathrm{LaBr}_{3}(\mathrm{Ce})$ (blue) energy spectra obtained by gating on the four background-subtracted transitions in the yrast band of ${ }^{110} R u$. (b) $E_{\gamma}-E_{\gamma}$ start-stop $\mathrm{LaBr}_{3}(\mathrm{Ce})$ matrix obtained from the same gates as (a). The left (white) and right (black) dots indicate the background gates used for the interpolation approach (all) and the three samples approach (encircled in red). The regions inside the black squares are the gates imposed on the $4_{1}^{+} \rightarrow 2_{1}^{+} \rightarrow 0_{g . s .}^{+}$. cascade.

Compton background produced by the $2_{1}^{+} \rightarrow 0_{\text {g.s. }}^{+}$and $4_{1}^{+} \rightarrow 2_{1}^{+}$transitions.

All gates indicated in Fig. 13(b) were used for the interpolation approach and the resulting fitted curves for the delayed and anti delayed $p \mid p^{m}$ time distributions are shown in Fig. 14(a) and (b), respectively. Different functions were used to fit the data points for the three components. Due to the small number of data points a second order polynomial was used for $\mathrm{C}_{p \mid b g}(\mathrm{E})$, while a curve of the form of Equation 18 was used for $\mathrm{C}_{b g \mid p}(\mathrm{E})$. The $\mathrm{C}_{b g \mid b g}(\mathrm{E})$ anti-delayed curve was observed to show an inversion of concavity when moving from the left to the right region of the coincidence peak, and therefore a third order polynomial was used in this case. Since the data points used for the $b g \mid b g$ re- 
gion are on the diagonal of the $\mathrm{E}_{\gamma}-\mathrm{E}_{\gamma}$ matrix, they can be plotted in Fig. 14 with respect to either of the two energy axis and here they were plotted for the lowest of the energy values defining the two-dimensional gate. This means that the value of $\mathrm{C}_{b g \mid b g}^{m}$ is obtained by taking the value $\mathrm{C}_{b g \mid b g}^{m}\left(\mathrm{E}^{d}\right)$. The other two background measurements $\mathrm{C}_{p \mid b g}^{m}$ and $\mathrm{C}_{b g \mid p}^{m}$ are obtained from $\mathrm{C}_{p \mid b g}\left(\mathrm{E}^{f}\right)$ and $\mathrm{C}_{b g \mid p}\left(\mathrm{E}^{d}\right)$, respectively. The measured delayed and anti-delayed $\mathrm{C}_{p \mid p}^{m}$ centroids and the centroids of the background obtained from the interpolation were then used in Eq. 15. The number of counts were measured from the regions indicated by the dots encircled in red in Fig. 13(b) and for the $\Pi$ ratio, defined as in Eq. 17, an average value of $\Pi \simeq 0.12$ was found between the delayed and anti-delayed coincidence peaks. The measured and "true" centroid positions, obtained from the interpolation approach, for the delayed and anti-delayed coincidence peaks and their background components are listed in the top half of Table 1 . The correction introduced by Eq. 16 is not identical for the two $p \mid p$ time distributions which therefore are not symmetric with respect to the $\Delta \mathrm{T}=0$ axis. This is due to statistical fluctuations which result from feeding Eq. 16 with very small $\Pi_{i}$ values which magnify any difference that statistically arises between the centroid of the delayed and anti-delayed distributions describing the same FEP or background component. The two corrected centroid positions $\mathrm{C}_{p \mid p}$ give a centroid difference of $\Delta \mathrm{C}=1158(76) \mathrm{ps}$, which together with a PRD correction of 192(6) ps give a lifetime value of $\tau=483(38)$ ps. Error bars for the centroid positions of the background components were obtained using $1 \sigma$ of confidence interval for the three fitted curves, in a procedure similar to the one described for the Compton curve.

For the three regions approach, two samples of each background component were taken by gating to the immediate left and right of the $241 \mathrm{keV}$ and $423 \mathrm{keV}$ peaks as shown in Fig. 13(b) by the six dots encircled in red. Each one of the six time distributions was first corrected for the Compton time-walk, then the centroids of the three background time distribution were calculated from the weighted average of the two time distributions describing each background component. The number of counts were calculated from the average of the number 
(a)

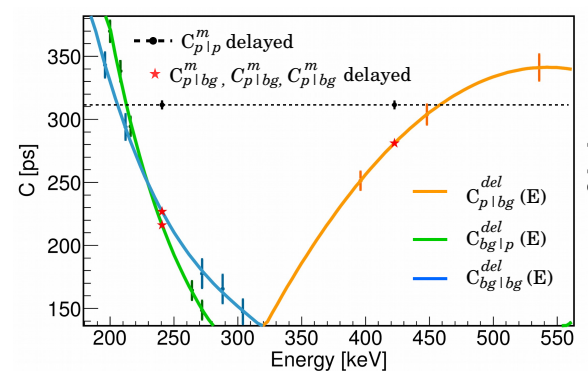

(b)

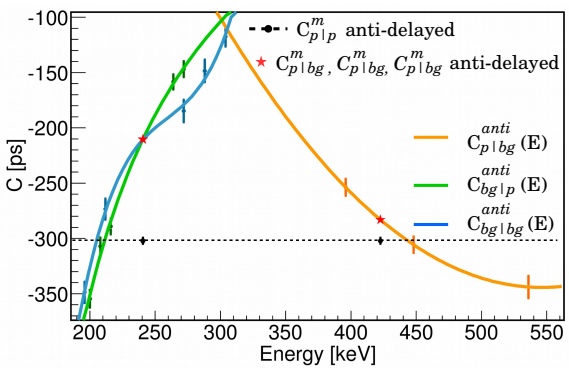

Figure 14: (a) Fitted curves describing the centroid of the background components of the delayed time distribution as a function of energy. The three red stars, indicating the values of the three curves at the energies $E^{d}$ and $E^{f}$ represent the $C_{p \mid b g}^{m}, C_{b g \mid p}^{m}$ and $C_{b g \mid b g}^{m}$ centroids. The centroid $C_{p \mid p}^{m}$ is indicated by the black dots at the two different energies $E^{d}$ and $E^{f}$. (b) Same as (a) but for the anti-delayed time distribution.

of counts of the two distributions measured for each background component, weighted according to the gate width. The centroid positions for the coincidence peak and background regions and their number of counts were then used in Eq. 15. The values used are listed in the bottom part of Table 1, both for the delayed and anti-delayed time distributions. It can be noticed that this time the effect of the scaling and the background correction gives symmetric results, with respect to $\Delta \mathrm{T}=0$, for the delayed and anti-delayed distributions. From $\Delta \mathrm{C}=1081(68)$ ps a lifetime of $\tau=445(34)$ ps was found using the three samples approach.

The lifetime values of $\tau=483(38)$ ps from the interpolation approach and $\tau=445(34)$ ps from the three samples approach agree within one standard deviation and, given the small peak-to-background ratio and the large error bars, they are remarkably consistent. The most recent evaluation for the value of lifetime of the $2^{+}$state in ${ }^{110} \mathrm{Ru}$, given in Ref. [19] is $\tau_{l}=462(29) \mathrm{ps}$, which is the unweighted average of the two measurements of $\tau=491(58)$ and $\tau=432(29)$ given in Ref. [20] and [21], respectively. 
Table 1: Measured and "true" centroid positions for the $p|p, p| b g, b g \mid p$ and bg $\mid b g$ regions, used to measure the lifetime of the $2^{+}$level in ${ }^{110}$ Ru together with the measured and "true" number of counts for the four regions. The results obtained from the interpolation method are listed in the top half of the table, while those from the three samples approach are in the bottom half. The measured $C^{m}$ centroids, for the background regions, have been shifted for the Compton time-walk. All the centroid positions are given in picoseconds.

\section{Interpolation}

\begin{tabular}{|c|c|c|c|c|c|c|c|}
\hline $\begin{array}{l}\dot{0} \\
\dot{0}\end{array}$ & $\begin{array}{c}\mathrm{C}_{p \mid p}^{m} \\
311(3) \\
\mathrm{n}_{p \mid p}^{m} \\
73661\end{array}$ & $\begin{array}{c}\mathrm{C}_{p \mid b g}^{m} \\
281(6) \\
\mathrm{n}_{p \mid b g}^{m} \\
53244\end{array}$ & $\begin{array}{c}\mathrm{C}_{b g \mid p}^{m} \\
216(4) \\
\mathrm{n}_{b g \mid p}^{m} \\
51771\end{array}$ & $\begin{array}{c}\mathrm{C}_{b g \mid b g}^{m} \\
227(5) \\
\mathrm{n}_{b g \mid b g}^{m} \\
40616\end{array}$ & $\begin{array}{c}\mathrm{C}_{p \mid b g}^{t} \\
455(26) \\
\mathrm{n}_{p \mid b g}^{t} \\
12628\end{array}$ & $\begin{array}{c}\mathrm{C}_{b g \mid p}^{t} \\
177(32) \\
\mathrm{n}_{b g \mid p}^{t} \\
11155\end{array}$ & $\begin{array}{c}\mathrm{C}_{p \mid p}^{t} \\
715(56) \\
\mathrm{n}_{p \mid p}^{t} \\
9262\end{array}$ \\
\hline 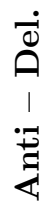 & $\begin{array}{c}\mathrm{C}_{p \mid p}^{m} \\
-302(3) \\
\mathrm{n}_{p \mid p}^{m} \\
71337\end{array}$ & $\begin{array}{c}\mathrm{C}_{p \mid b g}^{m} \\
-283(6) \\
\mathrm{n}_{p \mid b g}^{m} \\
51923\end{array}$ & $\begin{array}{c}\mathrm{C}_{b g \mid p}^{m} \\
-210(4) \\
\mathrm{n}_{b g \mid p}^{m} \\
50835\end{array}$ & $\begin{array}{c}\mathrm{C}_{b g \mid b g}^{m} \\
-211(5) \\
\mathrm{n}_{b g \mid b g}^{m} \\
440784\end{array}$ & $\begin{array}{c}\mathrm{C}_{p \mid b g}^{t} \\
-443(23) \\
\mathrm{n}_{p \mid b g}^{t} \\
11138\end{array}$ & $\begin{array}{c}\mathrm{C}_{b g \mid p}^{t} \\
-209(26) \\
\mathrm{n}_{b g \mid p}^{t} \\
10050\end{array}$ & $\begin{array}{c}\mathrm{C}_{p \mid p}^{t} \\
-443(52) \\
\mathrm{n}_{p \mid p}^{t} \\
9364\end{array}$ \\
\hline \multicolumn{8}{|c|}{ Three samples } \\
\hline 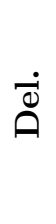 & $\begin{array}{c}\mathrm{C}_{p \mid p}^{m} \\
311(3) \\
\mathrm{n}_{p \mid p}^{m} \\
73661\end{array}$ & $\begin{array}{c}\mathrm{C}_{p \mid b g}^{m} \\
288(4) \\
\mathrm{n}_{p \mid b g}^{m} \\
53403\end{array}$ & $\begin{array}{c}\mathrm{C}_{b g \mid p}^{m} \\
222(3) \\
\mathrm{n}_{b g \mid p}^{m} \\
51024\end{array}$ & $\begin{array}{c}\mathrm{C}_{b g \mid b g}^{m} \\
218(7) \\
\mathrm{n}_{b g \mid b g}^{m} \\
39601\end{array}$ & $\begin{array}{c}\mathrm{C}_{p \mid b g}^{t} \\
490(23) \\
\mathrm{n}_{p \mid b g}^{t} \\
13802\end{array}$ & $\begin{array}{c}\mathrm{C}_{b g \mid p}^{t} \\
235(26) \\
\mathrm{n}_{b g \mid p}^{t} \\
11423\end{array}$ & $\begin{array}{c}\mathrm{C}_{p \mid p}^{t} \\
552(51) \\
\mathrm{n}_{p \mid p}^{t} \\
8835\end{array}$ \\
\hline 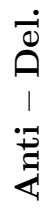 & $\begin{array}{c}\mathrm{C}_{p \mid p}^{m} \\
-302(3) \\
\mathrm{n}_{p \mid p}^{m} \\
71337\end{array}$ & $\begin{array}{c}\mathrm{C}_{p \mid b g}^{m} \\
-278(4) \\
\mathrm{n}_{p \mid b g}^{m} \\
51827\end{array}$ & $\begin{array}{c}\mathrm{C}_{b g \mid p}^{m} \\
-210(3) \\
\mathrm{n}_{b g \mid p}^{m} \\
49945\end{array}$ & $\begin{array}{c}\mathrm{C}_{b g \mid b g}^{m} \\
-210(6) \\
\mathrm{n}_{b g \mid b g}^{m} \\
40033\end{array}$ & $\begin{array}{c}\mathrm{C}_{p \mid b g}^{t} \\
-490(28) \\
\mathrm{n}_{p \mid b g}^{t} \\
11794\end{array}$ & $\begin{array}{c}\mathrm{C}_{b g \mid p}^{t} \\
-211(30) \\
\mathrm{n}_{b g \mid p}^{t} \\
9912\end{array}$ & $\begin{array}{c}\mathrm{C}_{p \mid p}^{t} \\
-529(44) \\
\mathrm{n}_{p \mid p}^{t} \\
9598\end{array}$ \\
\hline
\end{tabular}

\subsection{The $2^{+}$state in ${ }^{114} \mathrm{Pd}$}

The $2_{1}^{+}$state in ${ }^{114} \mathrm{Pd}$ has been isolated by setting background-subtracted gates on the $6_{1}^{+} \rightarrow 4_{1}^{+}(648 \mathrm{keV}), 8_{1}^{+} \rightarrow 6_{1}^{+}(715 \mathrm{keV}), 10_{1}^{+} \rightarrow 8_{1}^{+}(644 \mathrm{keV})$ and $5_{1}^{-} \rightarrow 4^{+}(1332 \mathrm{keV})$ transitions in Gammasphere. For this measurement the delayed and anti-delayed $p \mid p$ regions were very clean with no contaminants and a $\Pi$ ratio of $\simeq 0.33$, calculated using Eq. 17, was obtained for the two coincidence peaks. It was only possible to select very clean background regions next to the coincidence peaks due to the large number of contaminants and 

in Fig. 15(a) and by the black and white dots in Fig. 15(b). The measured and for each background component were selected, as indicated by the red solid lines

(a)

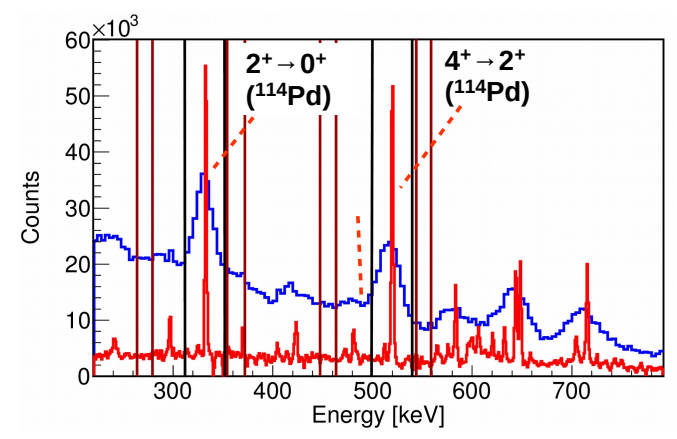

(b)

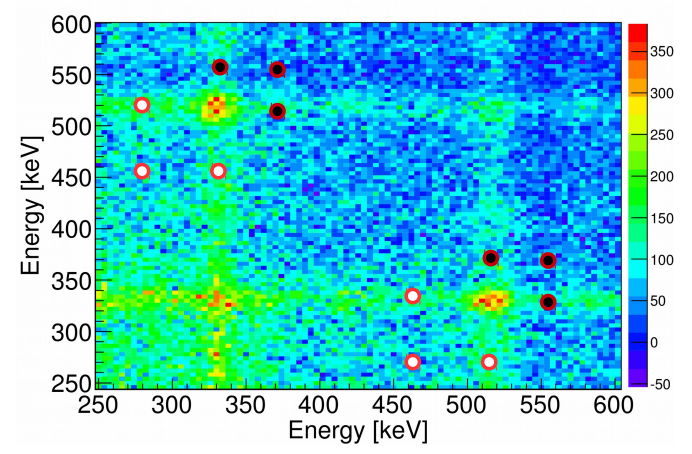

Figure 15: (a) HPGe (red) and $\mathrm{LaBr}_{3}$ (blue) energy spectra gated on the $6_{1}^{+} \rightarrow 4_{1}^{+}$, $8_{1}^{+} \rightarrow 6_{1}^{+}, 10_{1}^{+} \rightarrow 8_{1}^{+}$and $5_{1}^{-} \rightarrow 4^{+}$transitions in ${ }^{114} \mathrm{Pd}$. (b) $E_{\gamma}-E_{\gamma}$ start-stop $\mathrm{LaBr}_{3}$ matrix obtained from the same gates as (a). The left (white) and right (black) background gates are indicated by the dots encircled in red.

therefore only the three samples approach was used. As for ${ }^{110} \mathrm{Ru}$, two regions

corrected centroid positions for the $p \mid p$ and the three background components are listed in Table 2. It can be observed that this time, after the background correction, the "true" delayed and anti-delayed time distributions are symmetric with respect to $\Delta \mathrm{T}=0$, within one standard deviation, proving the effectiveness of the selected background regions. The same cannot be observed for the "true" centroid positions of the three background components: once again statistical fluctuations and relatively small $\Pi_{i}$ values are the reason for this difference. 
From the two $\mathrm{C}_{p \mid p}^{t}$ values, a centroid difference value of $\Delta \mathrm{C}=360(24)$ ps was found which, together with a PRD correction of 152(6) ps, gives a lifetime value of $\tau=104(12)$ ps. This is within one sigma of the literature value of $\tau_{l}=118(20)$ ps given in Ref. [22] and has a smaller error bar. It is also consistent with the value of $\tau=116(6)$ ps in Ref. [23] but never published.

Table 2: Same as Table 1, but for the $2^{+}$state in ${ }^{114} \mathrm{Pd}$ and for the three samples approach only.

\begin{tabular}{|c|c|c|c|c|c|c|c|}
\hline \multirow{4}{*}{$\dot{\oplus}$} & $\mathrm{C}_{p \mid p}^{m}$ & $\mathrm{C}_{p \mid b g}^{m}$ & $\mathrm{C}_{b g \mid p}^{m}$ & $\mathrm{C}_{b g \mid b g}^{m}$ & $\mathrm{C}_{p \mid b g}^{t}$ & $\mathrm{C}_{b g \mid p}^{t}$ & $\mathrm{C}_{p \mid p}^{t}$ \\
\hline & $130(2)$ & $137(4)$ & $89(5)$ & $110(9)$ & $193(18)$ & $61(25)$ & $175(17)$ \\
\hline & $\mathrm{n}_{p \mid p}^{m}$ & $\mathrm{n}_{p \mid b g}^{m}$ & $\mathrm{n}_{b g \mid p}^{m}$ & $\mathrm{n}_{b g \mid b g}^{m}$ & $\mathrm{n}_{p \mid b g}^{t}$ & $\mathrm{n}_{b g \mid p}^{t}$ & $\mathrm{n}_{p \mid p}^{t}$ \\
\hline & 15552 & 7395 & 8656 & 4930 & 2464 & 3725 & 4434 \\
\hline \multirow{4}{*}{ 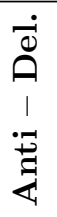 } & $\mathrm{C}_{p \mid p}^{m}$ & $\mathrm{C}_{p \mid b g}^{m}$ & $\mathrm{C}_{b g \mid p}^{m}$ & $\mathrm{C}_{b g \mid b g}^{m}$ & $\mathrm{C}_{p \mid b g}^{t}$ & $\mathrm{C}_{b g \mid p}^{t}$ & $\mathrm{C}_{p \mid p}^{t}$ \\
\hline & $-148(2)$ & $-140(4)$ & $-123(6)$ & $-129(11)$ & $-164(15)$ & $-116(16)$ & $-185(17)$ \\
\hline & $\mathrm{n}_{p \mid p}^{m}$ & $\mathrm{n}_{p \mid b g}^{m}$ & $\mathrm{n}_{b g \mid p}^{m}$ & $\mathrm{n}_{b g \mid b g}^{m}$ & $\mathrm{n}_{p \mid b g}^{t}$ & $\mathrm{n}_{b g \mid p}^{t}$ & $\mathrm{n}_{p \mid p}^{t}$ \\
\hline & 16024 & 7292 & 8805 & 5048 & 2243 & 3758 & 5048 \\
\hline
\end{tabular}

\section{Conclusion}

After an exhaustive description of the composition of the background characterising $\gamma-\gamma$ coincidence data, a new background subtraction method for the time information is suggested. This is summarised by Eq. 15 and, in order to be effective, it requires the precise determination of the apparent lifetime of three background components and the weight of their contribution to the measured time distribution.

The interpolation approach provides good results when several clean gates can be applied for each background component.

The three samples approach, which requires at least one background gate for each component, is useful when the number of contaminants is quite large and it is not possible to perform the number of fits required by the interpolation method. When using this approach, the centroids of the three background time distributions have to be corrected for the Compton time-walk. 
Both approaches are equally valid and give consistent results as proven by the three measurements presented on the lifetime of the $2_{1}^{+}$states in ${ }^{110} \mathrm{Ru}$ and

${ }^{114} \mathrm{Pd}$. Despite the very low $\Pi$ and $\Pi_{i}$ values, the measurements are consistent with the literature and, in the ${ }^{114} \mathrm{Pd}$ case, more accurate. The application of this background correction method is strongly suggested when dealing with difficult datasets but, because the logic behind it is quite stringent, we recommend its use in all cases where the background is not negligible.

\section{Acknowledgements}

The author E. R. G. would like to acknowledge the Science \& Technology Facility Council for funding his $\mathrm{PhD}$ studentship.

All the authors are grateful to the FATIMA \& Gammasphere collaboration, for providing the equipment used for the measurements presented in this work.

\section{References}

[1] H. Mach et al., Nuclear Physics A523 (1991) 197.

[2] W.D. Hamilton, The electromagnetic interaction in nuclear spectroscopy, North-Holland New York 1975, 173.

[3] H. Mach, R. L. Gill and M. Moszynski, Nuclear Instruments and Methods in Physics Research Section A 280, (1989) 49.

[4] M. Moszynski and H. Mach, Nuclear Instruments and Methods in Physics Research Section A 277, (1989) 407.

[5] N. Mărginean et al., European Physics Journal A 46 (2010) 329.

[6] J.-M Régis et al., Nuclear Instruments and Methods in Physics Research Section A 622 (2010) 83.

[7] J.-M Régis et al., Nuclear Instruments and Methods in Physics Research Section A 726 (2013) 191. 
[8] J.-M Régis et al., Nuclear Instruments and Methods in Physics Research Section A 897 (2018) 38.

[9] S. Ansari et al., Physical Review C 96 (2017), 054323.

[10] I-Y.Lee et al. Nuclear Physics A 520 (1990), 641c .

[11] L. M. Fraile et al. FATIMA Technical Design Report (2015).

[12] M. Rudigier et al. Acta Physica Polonica B 48, (2017) 351.

[13] G. Lorusso et al. European Physics Letters 120, (2017) 22001.

[14] M. Morháč et al., Nuclear Instruments and Methods in Physics Research Section A 401 (1997) 113.

[15] J.-M Régis et al., Nuclear Instruments and Methods in Physics Research Section A 684 (2012) 36.

[16] H. Mach et al., Physics Letters B 230, (1989) 21.

[17] L. M. Fraile et al. Nuclear Physics A 657 (1999), 355.

[18] J.-M Régis et al., Nuclear Instruments and Methods in Physics Research Section A 897 (2018) 38.

[19] G. Gürdal and F. G. Kondev, Nuclear Data Sheets 113, (2012) 1315.

[20] R. C. Jared, H. Nifenecker, S. G. Thompson, Proceedings for the 3rd Symposium Physics Chemistry Fission 2, (1974) 211.

[21] E. Cheifetz et al. Proc. Conf. Nucl. Spectr. Fission Products, 193 (1980).

[22] A. Dewald et al. Physical Review C 78, (2008) 051302.

[23] H. Mach et al. JYFL annual report, 2003. 